\title{
A Histopathology-based Assessment of Biological Behavior in Oral Hyalinizing Extraosseous Lesions by Differential Stains
}

\author{
Aamir M Nadeem ${ }^{1}$, Bhaskar Nagaraj ${ }^{2}$, Deepak A Jagadish ${ }^{3}$, Dhruv Shetty ${ }^{4}$, Surendra Lakshminarayana ${ }^{5}$, Dominic Augustine ${ }^{6}$,
} Roopa S Rao ${ }^{7}$

\begin{abstract}
Aim: The assessment of hyalinization to determine aggressive behavior in oral pathological lesions is a scarcely researched field that requires further exploration. The current study aims to predict the biological behavior of oral hyalinizing extraosseous lesions (OHEOL) by employing four differential stains with clinicopathologic correlation.

Materials and methods: The study was performed on retrospectively diagnosed formalin-fixed paraffin-embedded cases of salivary gland tumors (SGTs) $(n=13)$, benign soft tissue (BST) lesions $(n=24)$, and oral submucous fibrosis (OSMF) $(n=53)$. The hematoxylin and eosin-stained sections were analyzed for the severity of hyalinization (SOH). Differential stains periodic acid Schiff (PAS), Alcian blue, safranin $\mathrm{O}$, and picrosirius red with polarizing microscopy were used to assess the components of hyalinized tissue. The SOH was correlated with differential staining characteristics and clinicopathologic features to analyze possible correlation with aggressive potential in BST, advancement of disease in OSMF, and recurrence in SGT.

Results: Intensity of picrosirius red stain significantly correlated with SOH of SGTs $(p=0.044)$. The intensity of PAS stain ( $p=0.040)$, picrosirius red polarizing greenish-yellow color $(p=0.002)$, and pattern of distribution of picrosirius red $(p=0.023)$ significantly correlated with recurrence of SGTs. The intensity of differential stains increased with the SOH in BST lesions indicating their correlation with SOH. The intensity $(p=0.008)$ and pattern $(p=0.010)$ of Alcian blue staining and intensity of safranin O stain $(p=0.003)$ significantly correlated with $\mathrm{SOH}$ in OSMF. Picrosirius red polarizing color reddish and yellowish red $(p=0.002)$ significantly correlated with SOH distinguishing early and advanced OSMF.

Conclusion: Picrosirius red and PAS stains are reliable indicators of $\mathrm{SOH}$ and recurrence potential in SGT. Alcian blue, safranin $\mathrm{O}$, and picrosirius red polarizing colors enable detection of $\mathrm{SOH}$ and accurately distinguish early from advanced OSMF.

Clinical significance: $\mathrm{SOH}$ can be considered as a histological predictor of aggressive biologic behavior in OHEOL. These findings will result in appropriate management protocols.

Keywords: Alcian blue, Fibrosis, Hyaline, PAS, Picrosirius red, Polarizing microscopy, Safranin O, Salivary gland neoplasms.

The Journal of Contemporary Dental Practice (2021): 10.5005/jp-journals-10024-3122
\end{abstract}

\section{INTRODUCTION}

Hyalinization is defined as the process of deposition of eosinophilic, structureless, homogeneous material. It is a process of modifying the internal and/or external environment of the cell and is recognized as an interaction between cellular matrix and acellular matrix. The presence of hyalinization in extraosseous lesions of the oral cavity has invoked considerable interest to analyze its presence and determine its influence on biology behavior. Oral hyalinizing extraosseous lesions (OHEOL) are diverse with varied behavior ranging from benign soft tissue (BST) tumors, locally aggressive benign lesions, oral potentially malignant disorders, and salivary gland tumors (SGTs).

However, the significance of hyalinization in oral benign and malignant lesions is still unclear as no comprehensive studies linking the intensity of hyalinization of the proteoglycan and hyaluronic acid content to the biological behavior of these lesions have been performed. Hyalinized collagen is characterized by an increase in polysaccharide hyaluronan. ${ }^{1}$ The concentration of hyaluronan is said to correlate with the intensity of hyalinization;, ${ }^{2,3}$ however, this still requires further investigation in tissue samples. Further, the role of hyalinization and its implications on the biologic behavior of OHEOL has not been explored to date.

\footnotetext{
${ }^{1-7}$ Department of Oral Pathology and Microbiology, Faculty of Dental Sciences, Ramaiah University of Applied Sciences, MSR Nagar, Bengaluru, Karnataka, India
}

Corresponding Author: Surendra Lakshminarayana, Department of Oral Pathology and Microbiology, Faculty of Dental Sciences, Ramaiah University of Applied Sciences, MSR Nagar, Bengaluru, Karnataka, India, Phone: +91 99804244187, e-mail: drsuri29@gmail.com

How to cite this article: Nadeem AM, Nagaraj B, Jagadish DA, et al. A Histopathology-based Assessment of Biological Behavior in Oral Hyalinizing Extraosseous Lesions by Differential Stains. J Contemp Dent Pract 2021;22(7):812-828.

Source of support: Nil

Conflict of interest: None

Colorimetric techniques labeled avidin-biotin method and spectrophotometry are expensive methods to detect hyalinization, they need expensive equipment and are time-consuming. ${ }^{4}$ The application of special stains is absolutely necessary; since it requires fewer types of equipment, interpretation is easy, rapid, and economical. ${ }^{5}$ The special stains we have chosen for the current study include PAS, Alcian blue, safranin $\mathrm{O}$, and picrosirius red with 
polarizing microscopy; these stains have staining characteristics that will provide an insight into the levels of proteoglycan content and the degree of hyalinization of OHEOL.

The minimal knowledge on the behavior of these lesions equates to a variety of schools of thought on their management ranging from unnecessarily aggressive to ineffective conservative treatment. The present study aims to evaluate the biological behavior of OHEOL through special stains by comparing severity of hyalinization $(\mathrm{SOH})$ with aggressive behavior. The $\mathrm{SOH}$ was established by estimating the staining intensity and distribution of PAS, picrosirius red, safranin $\mathrm{O}$, and Alcian blue, the $\mathrm{SOH}$ was correlated with clinicopathological parameters of OHEOL to determine a possible correlation. The histologic feature of hyalinization was evaluated for the first time through this study in OHEOL with clinicopathologic correlation.

\section{Methodology}

Ethical clearance to undertake the study was obtained from the M.S. Ramaiah University of Applied Sciences (RUAS) Ethics Committee. The study groups considered under the category of soft tissue lesions of the oral cavity were SGTs $(n=13)$, oral submucous fibrosis (OSMF) $(n=53)$, and BSTs $(n=24)$. The formalin-fixed paraffin-embedded blocks of the cases were obtained from the archives of the Department of Oral Pathology and Microbiology, Faculty of Dental Sciences, M.S. RUAS. The SGTs were further subdivided into benign and malignant, pleomorphic adenomas $(n=5)$, carcinoma ex pleomorphic adenoma $(n=6)$, and mucoepidermoid carcinoma $(n=2)$. The OSMF cases considered comprised of early OSMF $(n=16)$ and late OSMF $(n=37)$. The BST group comprised of 13 reactive lesions-irritation fibroma $(n=12)$ and traumatic neuroma $(n=1)$ and 11 tumor/tumor-like lesions-schwannoma $(n=4)$, nodular fasciitis $(n=3)$, and myxoid variant of neurofibroma $(n=4)$. The archival tissues were retrieved and deparaffinized at $57^{\circ} \mathrm{C}$ for 10 minutes for the wax to melt; the remaining wax was removed using xylene. From the archival clinical files, descriptive data and clinical parameters were noted and tabulated. The specimens were stained using four special stains-PAS, safranin O, Alcian blue, and picrosirius red using the following protocols:

- PAS Staining Protocol ${ }^{6}$

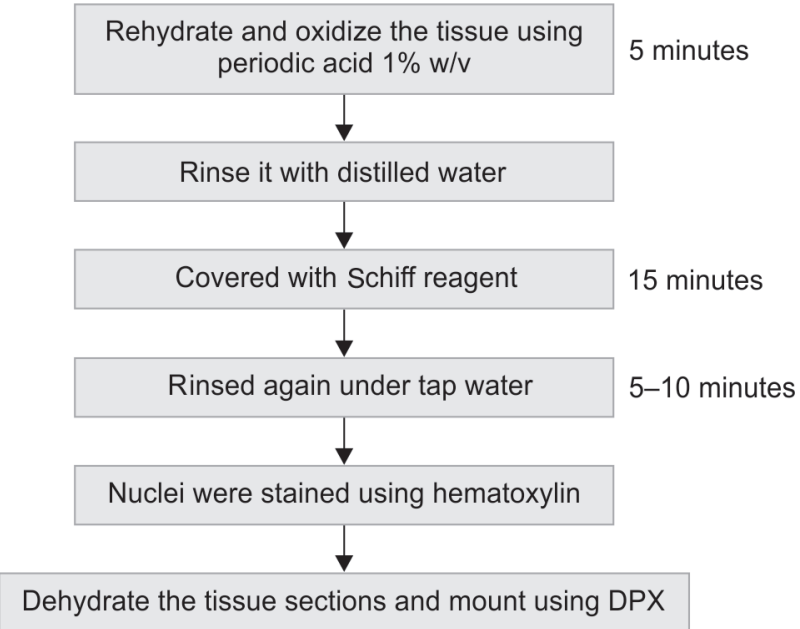

- Safranin O Staining Protocol ${ }^{7}$

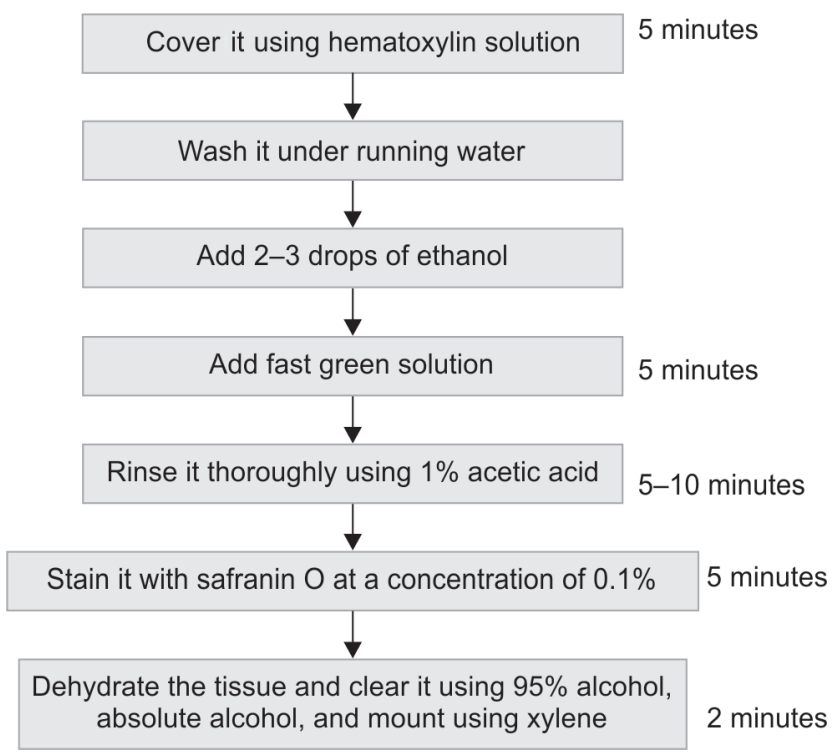

- Alcian blue Staining Protocol ${ }^{8}$

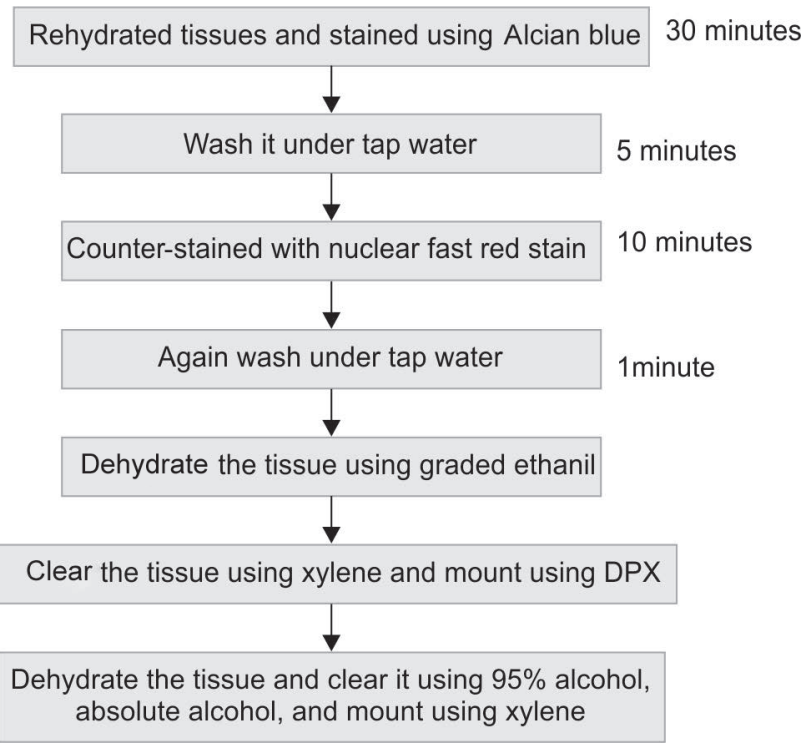

- Picrosirius Red Staining Protocol ${ }^{9}$

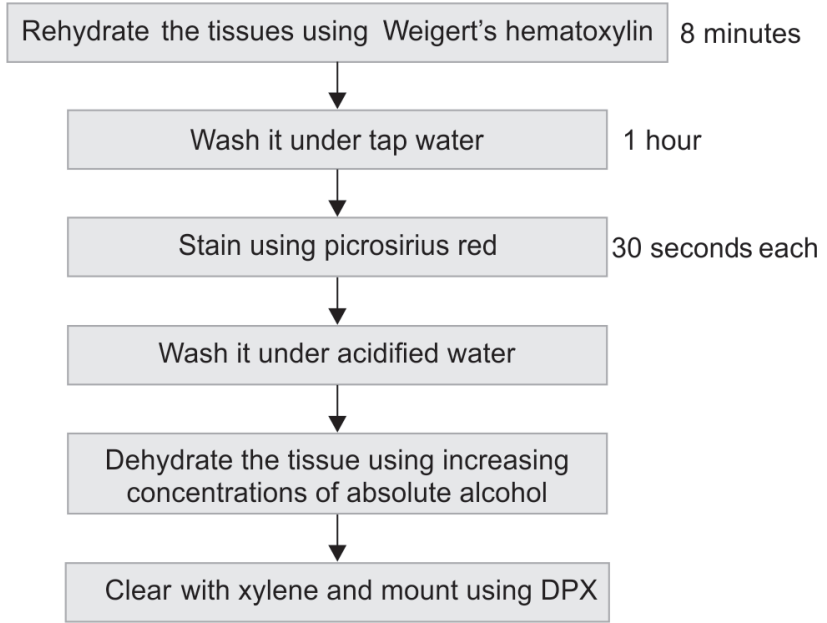




\section{Histopathological and Clinicopathologic Correlation of OHEOL with Hyalinization and Recurrence}

\section{SGT Group}

The clinical parameters of the site and bone erosion were analyzed for statistical correlation with $\mathrm{SOH}$ and recurrence. The staining intensity and distribution pattern of special stains were analyzed based on histological parameters and were correlated with the $\mathrm{SOH}$ and recurrence of SGTs. The subdivision of benign and malignant lesions of SGTs was correlated with recurrence and $\mathrm{SOH}$. This was done to clearly understand the relationship of individual SGT lesions with $\mathrm{SOH}$ and recurrence.

\section{OSMF Group}

General parameters of the site, atrophic epithelium, and muscle degeneration were analyzed for statistical correlation with both $\mathrm{SOH}$ and recurrence. Histological parameters of special staining features were also assessed for possible correlation with $\mathrm{SOH}$ in OSMF.

\section{BST Group}

The clinical parameters of the site, size, and bone erosion were analyzed for statistical correlation with $\mathrm{SOH}$. The histological parameters of special staining features (intensity of staining and distribution pattern) were assessed for possible correlation with $\mathrm{SOH}$ and aggressive biologic behavior of OHEOL.

\section{Interpretation, Scoring, and Statistical Analysis}

Special stained sections were examined for histological parameters by two oral pathologists (D.A. and L.S.); in case of uncertainty, a third oral pathologist R.S.R. examined the slide. In $\mathrm{H}$ \& E-stained slides, the $\mathrm{SOH}$ was recorded as $0=$ absent, $1=$ mild, $2=$ moderate, and 3 = intense. For PAS, safranin $\mathrm{O}$, and Alcian blue, scoring criteria were scored as $1=$ mild, $2=$ moderate, and $3=$ intense. For scoring assessment for the pattern of distribution, $0=$ not significant (NS), $1=$ focal, and $2=$ diffuse. For the assessment of picrosirius red under polarizing microscopy, the same scoring criteria were followed. The scoring criteria for polarizing birefringent colors utilized were 1 = greenish, 2 = greenish-yellow, $3=$ reddishyellow, and $4=$ reddish. The photomicrographs of slides were taken at $\times 100$ magnification using a Jenoptik Progres Gryphax Arktur USB 3.0 microscope camera, Jena, Germany mounted on an Olympus Optical Microscope BX53F2, Tokyo, Japan. The clinicopathological and histopathological parameter scores were recorded and statistically analyzed using chi-square test, keeping $p<0.05$ as a significant value. The statistical analysis was done using Statistical Package for the Social Sciences (SPSS) software version 20.0, International Business Machines Corporation, New York, New York, USA.

\section{Results}

\section{SGT Group}

\section{Correlation of Clinical Parameters with SOH and Recurrence}

The descriptive features of SGT cases $(n=13)$ are shown in Figure 1. When the clinical parameters of site and bone erosion were analyzed for statistical correlation with $\mathrm{SOH}$, all parameters were found to be statistically nonsignificant. When the same features were analyzed for statistical correlation with recurrence, location in the posterior palate involving pterygoid plate $(p=0.027)$ and diffuse erosion ( $p=0.006$ ) favored recurrence (Table 1).
Table 1: Comparison of correlation of clinical parameters with recurrence of SGT

\begin{tabular}{|c|c|c|c|c|c|}
\hline \multirow{2}{*}{\multicolumn{2}{|c|}{ Clinical parameters }} & \multicolumn{2}{|c|}{ Recurrence } & \multirow[b]{2}{*}{$x^{2}$} & \multirow[b]{2}{*}{$\begin{array}{c}p \\
\text { value }\end{array}$} \\
\hline & & $\begin{array}{c}\text { Absent } \\
\text { (\%) }\end{array}$ & $\begin{array}{c}\text { Present } \\
\text { (\%) }\end{array}$ & & \\
\hline \multirow[t]{3}{*}{ Site } & Buccal mucosa & 100 & 0 & \multirow[b]{3}{*}{7.252} & \multirow[b]{3}{*}{0.027} \\
\hline & Palate & 71.4 & 28.6 & & \\
\hline & $\begin{array}{l}\text { Palate and } \\
\text { pterygoid } \\
\text { plates }\end{array}$ & 0 & 100 & & \\
\hline \multirow{3}{*}{$\begin{array}{l}\text { Radiographic } \\
\text { features }\end{array}$} & No involvement & 100 & 0.0 & \multirow{3}{*}{10.317} & \multirow{3}{*}{0.006} \\
\hline & $\begin{array}{l}\text { Bone erosion } \\
\text { with sclerotic } \\
\text { border }\end{array}$ & 33.3 & 66.7 & & \\
\hline & $\begin{array}{l}\text { Diffuse bone } \\
\text { erosion }\end{array}$ & 0 & 100 & & \\
\hline
\end{tabular}

Chi-squared test, $p$-value $<0.05$-statistically significant

\section{Correlation of Histological Parameters with $\mathrm{SOH}$ and Recurrence}

PAS, Alcian blue, and Safranin O Special Stain Analysis:

When the intensity and pattern of distribution of the special stains were correlated with SOH of SGTs, it was observed that the intensity of staining and pattern of distribution of PAS, Alcian blue, and safranin $\mathrm{O}$ did not show any significant statistical correlation with $\mathrm{SOH}$. When the same was correlated with the recurrence of SGTs, it was observed that the intensity of PAS $(p=0.040)$ showed a significant statistical correlation with recurrence. The pattern of distribution was found to be statistically insignificant (Table 2 and Fig. 2).

\section{Picrosirius Red Polarizing Microscopy Special Stain Analysis:}

When conventional and polarizing microscopy was used to analyze intensity, pattern of distribution, and the polarizing colors, it was evident that staining intensity of Picrosirius red stain significantly correlated with SOH of SGTs ( $p=0.044$ ) (Table 3$)$. It was noted that polarizing greenish-yellow color $(p=0.002)$ and pattern of distribution (absence of a diffuse pattern) $(p=0.023)$ significantly correlated with the recurrence of SGTs (Table 2).

\section{Correlation of SOH with Recurrence in SGTs}

When the SOH was correlated with recurrence in SGT's subgroups, it was observed that $\mathrm{SOH}$ strongly correlated with recurrence $(p=0.034)$. Irrespective of the benign or malignant nature of the neoplasm the $\mathrm{SOH}$ was found to correlate significantly with the recurrence potential of the lesion (Table 4 and Fig. 3).

\section{BST Group}

\section{Correlation of Clinical Parameters with $\mathrm{SOH}$}

The descriptive features of BSTs cases $(n=24)$ are shown in Table 5. When the clinical parameters of the site, size, and bone erosion were analyzed for statistical correlation with $\mathrm{SOH}$ it was noted that the features of location in the mandibular-maxillary vestibule, corner of the mouth, and gingiva correlated significantly with $\mathrm{SOH}$ $(p=0.000)$.

\section{Correlation of Histological Parameters with $\mathrm{SOH}$}

When the histological parameters of special staining (intensity of staining and distribution pattern of PAS, Alcian blue, safranin $\mathrm{O}$, 


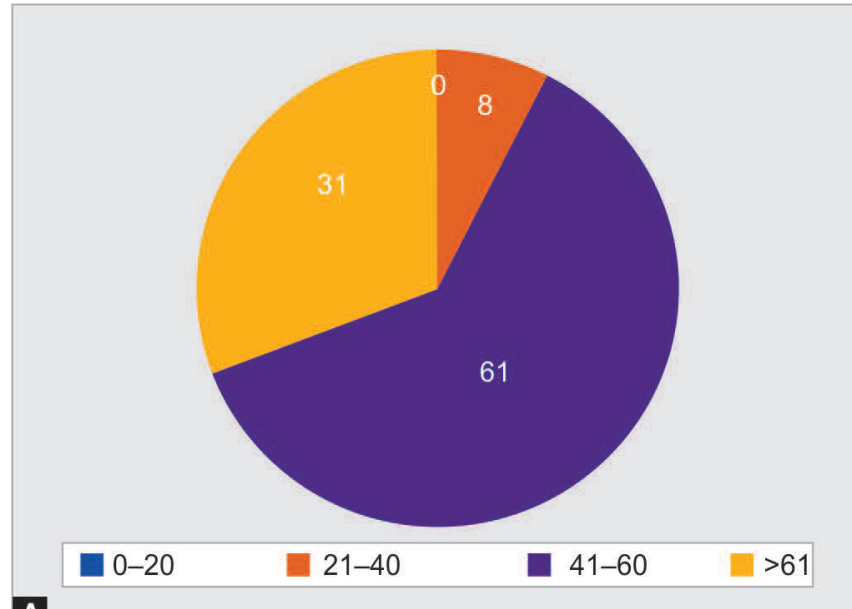

A
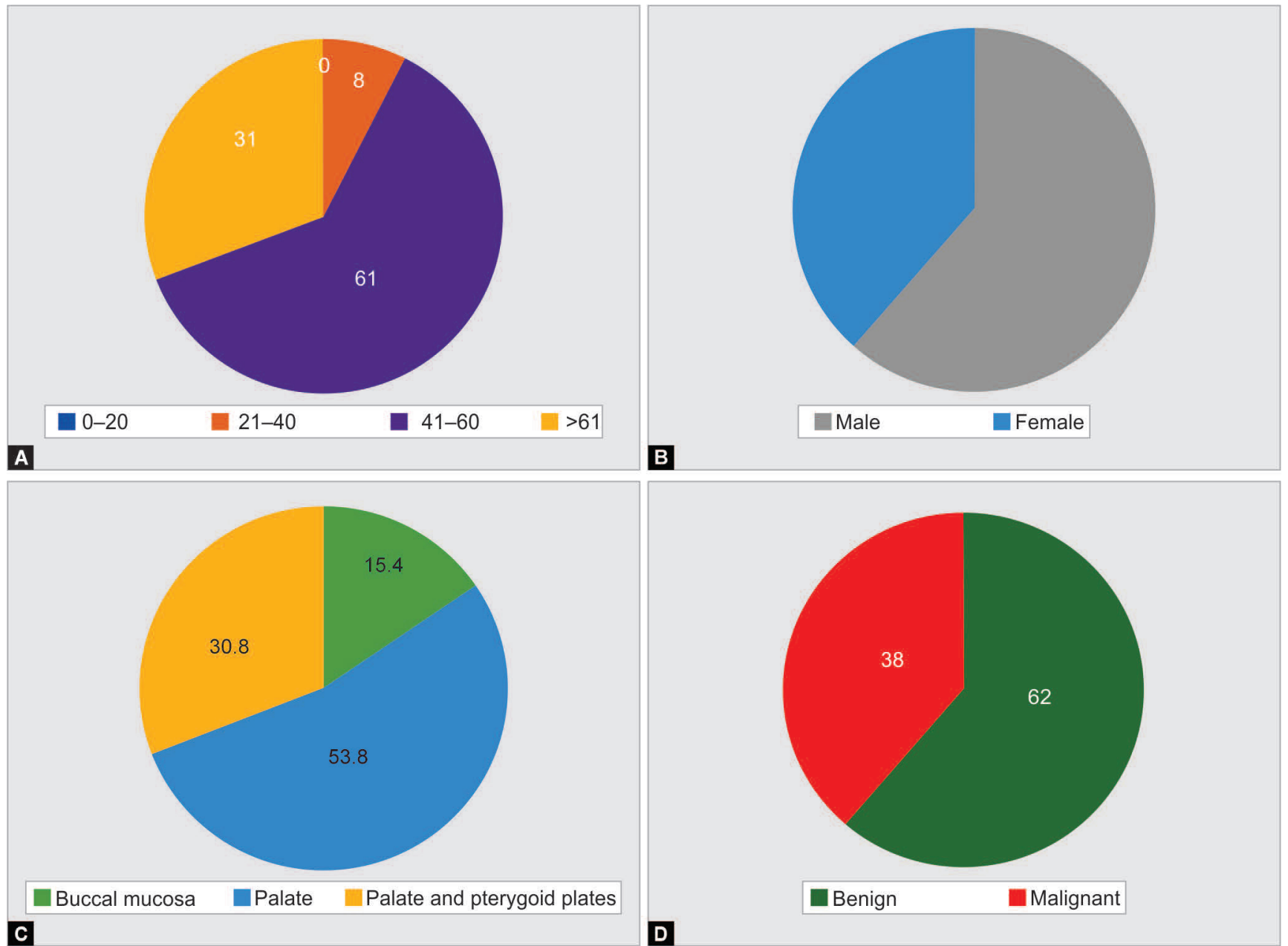

Figs 1 A to D: Descriptive features of salivary gland tumor cases. (A) Age groups; (B) Gender; (C) Sub-groups; (D) Site

picrosirius red polarizing microscopy) were assessed for possible correlation with $\mathrm{SOH}$ and aggressive biologic behavior of OHEOL, no statistical correlation was observed. However, it was evident that the intensity of differential stains increased with the $\mathrm{SOH}$ implicating that these differential stains do show a correlation with $\mathrm{SOH}$ even though statistically not significant. The distinction in $\mathrm{SOH}$ between reactive lesions and tumor/tumor-like lesions is indicated in Figure 4.

\section{OSMF Group}

\section{Correlation of General Parameters with $\mathrm{SOH}$}

The descriptive features of OSMF cases $(n=53)$ are shown in Figure 5. When the general parameters of the site, atrophic epithelium, and muscle degeneration were analyzed for statistical correlation with $\mathrm{SOH}$, it was noted that atrophic epithelium $(p=0.001)$ and muscle degeneration ( $p=0.000$ ) showed a strong statistical correlation with SOH (Table 6).

\section{Correlation of Histological Parameters with $\mathrm{SOH}$}

When the histological parameters of special staining (intensity of staining and distribution pattern of PAS, Alcian blue, safranin O, picrosirius red polarizing microscopy) were assessed for possible correlation with $\mathrm{SOH}$, it was observed that intensity $(p=0.008)$ and pattern ( $p=0.010$ ) of Alcian blue staining and intensity of safranin O stain ( $p=0.003$ ) significantly correlated with $\mathrm{SOH}$. Picrosirius red polarizing color reddish and yellowish red $(p=0.002)$ significantly correlated with $\mathrm{SOH}$ enabling to distinguish early and advanced OSMF (Table 7 and Fig. 6).

\section{Discussion}

Hyalinized collagen in benign and malignant lesions is known to influence the biological behavior of neoplasms. ${ }^{10}$ The precise significance of this phenomenon in pathologic conditions has been inadequately researched. Recent studies have postulated the influence of collagen type on the biological behavior of oral neoplasms. ${ }^{11}$ Lately, the association of hyalinization and biologic behavior of oral diseases has been brought to light about odontogenic keratocyst, ${ }^{12}$ this has invoked considerable interest in further exploring the association of hyalinization in OHEOL. A cost-effective yet reliable method to detect hyalinization in tissues is by utilizing differential stains.

The current study attempts to identify these products in hyalinized tissue and to correlate it with clinical and 
Table 2: Comparison of correlation of histological parameters with recurrence of SGT

\begin{tabular}{|c|c|c|c|c|c|}
\hline & & \multicolumn{2}{|c|}{ Recurrence } & \multirow[b]{2}{*}{$x^{2}$} & \multirow[b]{2}{*}{$\begin{array}{c}p \\
\text { value }\end{array}$} \\
\hline \multicolumn{2}{|c|}{ Histological parameters } & $\begin{array}{c}\text { Absent } \\
\text { (\%) }\end{array}$ & $\begin{array}{c}\text { Present } \\
\text { (\%) }\end{array}$ & & \\
\hline \multirow{3}{*}{$\begin{array}{l}\text { PR } \\
\text { color }\end{array}$} & Reddish & 100.0 & 0.0 & & \\
\hline & Yellowish-red & 100.0 & 0.0 & 13.000 & 0.002 \\
\hline & Greenish-yellow & 10.0 & 100.0 & & \\
\hline \multirow{3}{*}{$\begin{array}{l}\text { PR } \\
\text { intensity }\end{array}$} & Mild & 0.0 & 0.0 & & \\
\hline & Moderate & 0.0 & 0.0 & - & - \\
\hline & Intense & 53.8 & 46.2 & & \\
\hline \multirow{3}{*}{$\begin{array}{l}\text { PR } \\
\text { pattern }\end{array}$} & NS & 0.0 & 100.0 & & \\
\hline & $F$ & 50.0 & 50.0 & 7.539 & 0.023 \\
\hline & D & 85.7 & 14.3 & & \\
\hline \multirow{3}{*}{$\begin{array}{l}\mathrm{AB} \\
\text { intensity }\end{array}$} & Mild & 0.0 & 0.0 & & \\
\hline & Moderate & 100 & 0.0 & 2.026 & 0.155 \\
\hline & Intense & 45.5 & 54.5 & & \\
\hline \multirow{3}{*}{$\begin{array}{l}\text { AB } \\
\text { pattern }\end{array}$} & NS & 0.0 & 0.0 & & \\
\hline & $F$ & 50.0 & 50.0 & 0.014 & 0.906 \\
\hline & D & 54.5 & 45.5 & & \\
\hline \multirow{3}{*}{$\begin{array}{l}\text { SNO } \\
\text { intensity }\end{array}$} & Mild & 0.0 & 0.0 & & \\
\hline & Moderate & 0.0 & 0.0 & - & - \\
\hline & Intense & 53.8 & 46.2 & & \\
\hline \multirow{3}{*}{$\begin{array}{l}\text { SNO } \\
\text { pattern }\end{array}$} & NS & 0.0 & 0.0 & & \\
\hline & $\mathbf{F}$ & 0.0 & 0.0 & - & - \\
\hline & D & 53.8 & 46.2 & & \\
\hline \multirow{3}{*}{$\begin{array}{l}\text { PAS } \\
\text { intensity }\end{array}$} & Mild & 100.0 & 0.0 & & \\
\hline & Moderate & 16.7 & 83.3 & 6.428 & 0.040 \\
\hline & Intense & 80.0 & 20.0 & & \\
\hline \multirow{3}{*}{$\begin{array}{l}\text { PAS } \\
\text { pattern }\end{array}$} & NS & 0.0 & 0.0 & & \\
\hline & $F$ & 0.0 & 0.0 & - & - \\
\hline & D & 53.8 & 46.2 & & \\
\hline
\end{tabular}

Chi-squared test, $p$-value $<0.05$-statistically significant

histopathological parameters to predict aggressive biologic behavior in OHEOL by using four special stains. Differential stains are employed to identify and demonstrate structures and tissues which are not visualized by routine $\mathrm{H} \& \mathrm{E}$ stain. Special stains are the preferred substitutes for expensive, time-consuming, and skilled-based techniques like immunohistochemistry. ${ }^{13}$ PAS is usually employed in histological interpretation, as it indicates the presence of carbohydrate compounds like glycogen and polysaccharide mucin. ${ }^{14}$ Picrosirius red is mainly employed to identify the collagen networks in different pathological tissues. Using polarized microscopy collagen appears red, yellow, or green with a dark background, this enables assessment of collagen type and maturity. ${ }^{15}$ Safranin $O$ is used to identify mucins and proteoglycans. ${ }^{16}$ Alcian blue is utilized in clinical histology to identify acid mucins, glycosaminoglycans, and hyaluronic acid. ${ }^{17}$ The above-mentioned connective tissue products are produced extensively in hyalinized connective tissue. The study groups considered under OHEOL include SGT, BST, and OSMF.
SGTs illustrate a varying range of benign and malignant tumors with diverse biological behavior. Their histologic diversity and varied biologic activity of tumoral cells make predicting their prognosis complex. ${ }^{18}$ Few studies have postulated that hyalinized stroma in SGTs favors aggressive behavior or malignant transformation of benign SGTs. ${ }^{19}$ Ellis and Auclair evaluated 65 cases of mixed SGTs for the atypical features of hypercellularity, capsule breach, hyalinization, necrosis, and cellular anaplasia; they concluded that benign mixed tumors that showed prominent zones of hyalinization were more likely to develop carcinoma. ${ }^{20}$

An extensively hyalinized stroma is also seen in hyalinizing clear cell carcinoma which is a rare minor SGT made up of clear cells and nests. The overall prognosis is good; however, few cases of occasional metastatic spread have been reported..$^{21}$ In adenoid cystic carcinoma (ACC) variably sized, often large, acellular hyaline matrix globules are characteristically noted, it is hypothesized that hyalinization in ACC is derived from abundant production of basal lamina by neoplastically modified myoepithelial cells and basaloid tumor epithelial cells or degeneration of the adjacent connective tissue. ${ }^{22,23}$ PAS intensely stains this hyalinized material magenta color. Polymorphous low-grade adenocarcinoma may produce a myxohyaline matrix similar to that of ACC and other basaloid SGTs. The epithelial-myoepithelial carcinoma also demonstrates variable hyaline sclerosis. ${ }^{24}$

Another study by lto et al. studied 189 pleomorphic adenomas and found hyalinization of the stroma in 22 cases (11.6\%). ${ }^{25}$ The authors stated that prominent zones of hyalinization have been related to aggressive behavior or malignant transformation of pleomorphic adenomas.

In the present study, extensive hyalinization was noted in carcinoma ex pleomorphic adenoma. It was observed that the intensity of PAS $(p=0.040)$ and picrosirius red stain $(p=0.044)$ showed a significant statistical correlation with the recurrence of SGT. It was noted that the intensity of picrosirius red stain significantly correlated with SOH of SGTs $(p=0.044)$. It was noted that polarizing greenish-yellow color $(p=0.002)$ and its pattern of distribution ( $p=0.023$ ) significantly correlated with the recurrence of SGTs. Overall, the SOH strongly correlated with recurrence for both benign and malignant SGT ( $p=0.034$ ) (Figs 7 and 8). Clinicopathologic features of location in the posterior palate involving pterygoid plate $(p=0.027)$ and diffuse erosion ( $p=0.006$ ) favored recurrence as lesions present posteriorly involving the pterygoid plate are usually detected late; added to this, diffuse erosion of the bone may indicate bone involvement that could worsen the prognosis. The results indicate the association of hyalinization with aggressive biologic behavior in SGTs.

BST tumors of the oral cavity are classified based on their cell of origin. ${ }^{26}$ Their biologic behavior varies drastically from an innocuous fibroma to the aggressive neurofibroma or locally destructive nodular fasciitis. The present study analyzed 24 BST lesions comprising of reactive lesions and tumor/tumor-like lesions to evaluate the role of hyalinized stroma in their biologic behavior.

When the histological parameters were assessed for possible correlation with $\mathrm{SOH}$ and aggressive biologic behavior of OHEOL, no statistical correlation was observed. This could be due to the small sample size and variable subgroups of BST considered 


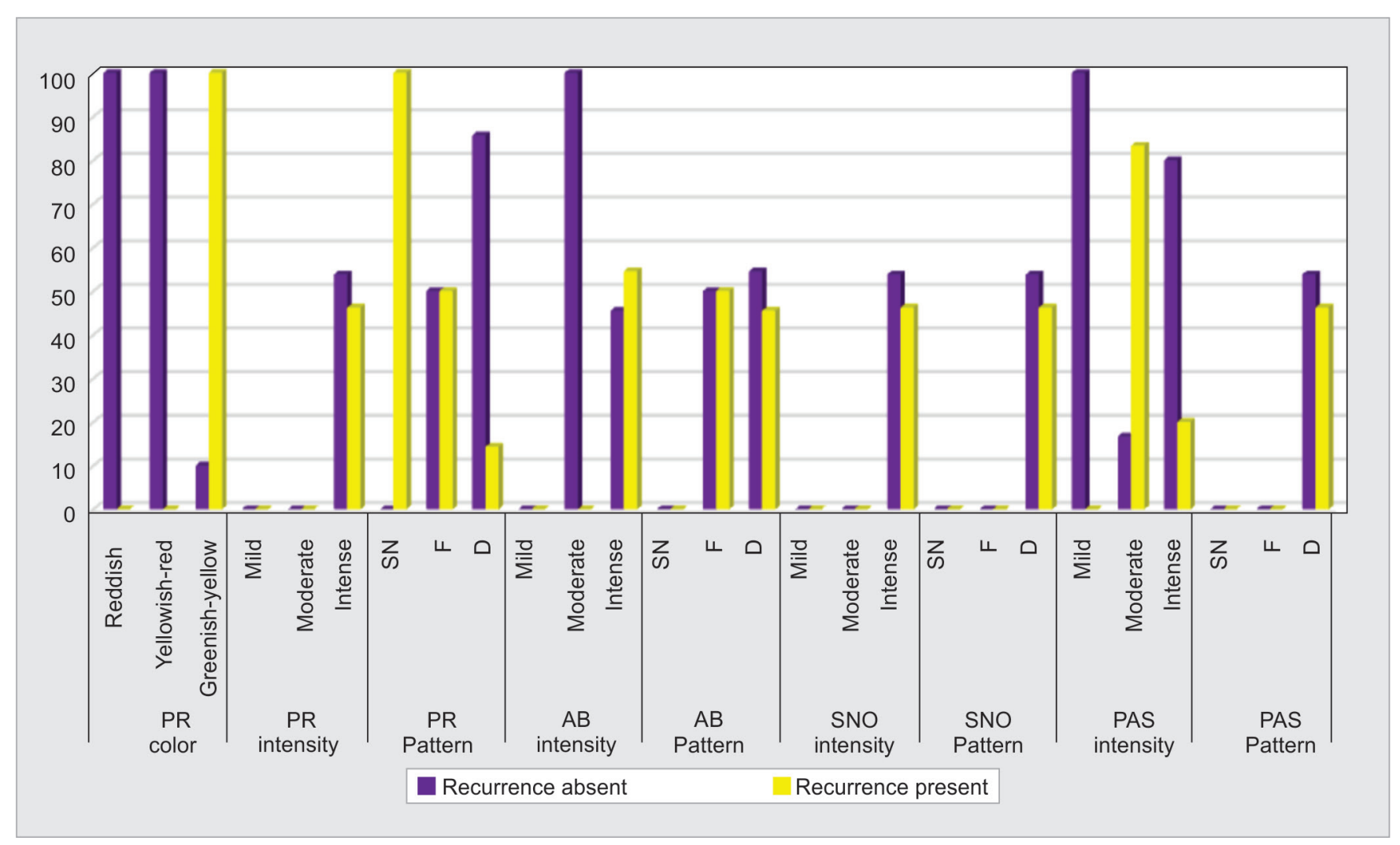

Fig. 2: Comparison of correlation of histological parameters with recurrence of SGT

in the current study. However, it is evident from the present study that the intensity of differential stains increased with the $\mathrm{SOH}$ implicating that these differential stains correlate well with $\mathrm{SOH}$ directly indicating that hyalinized BSTs possess an aggressive potential. This finding can be observed in Figure 5 which demonstrates intense staining by a myxoid variant of neurofibroma and nodular fasciitis for differential stains in contrast to mild staining observed in traumatic neuroma and irritational fibroma. A contrasting difference in polarizing colors can also be observed between the aggressive and nonaggressive BST lesions (Fig. 9).

To further substantiate this finding, a convincing hypothesis has been proposed by Chouaib et al., to explain the link between hyalinization and aggressive potential in soft tissue tumors. The authors stated that hyalinization leads to hypoxia promoting increased vascular endothelial growth factor (VEGF) production in tumor cells resulting in angiogenesis, especially at the invading front. As the tumor enlarges, there is vascular encroachment at the advancing front leading to hyalinized vascular regions, endothelial obliteration, tumor hypoxia, and more VEGF production, this vicious cycle leads to aggressive biologic behavior in soft tissue tumors. ${ }^{27}$

OSMF is a potentially malignant oral disorder with increased deposition of collagen (fibrosis) that is pathognomonic of the condition. ${ }^{28}$ The earliest reported histological studies on OSMF were by Pindborg and coworkers who reported increased dense collection of collagen fibers, juxta-epithelial hyalinization in advanced cases, variable inflammatory infiltration, edema, and decreased vascularity with advancing grades. These have

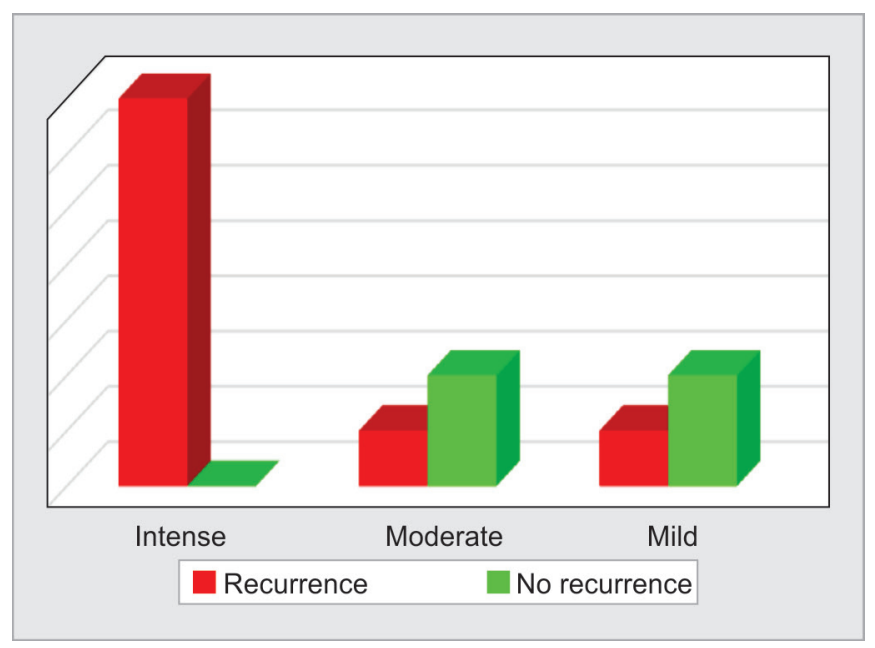

Fig. 3: Comparison of correlation of SOH with recurrence of SGT

largely been corroborated in subsequent reports except for juxta-epithelial hyalinization. This feature seems to be intensely variable and only a few cases of advanced OSMF have been reported with this finding. ${ }^{29}$ The hyalinization in OSMF is absent in the early stages of the disease. However, the assessment of hyalinization by a panel of differential stains has seldom been attempted. 
Table 3: Comparison of correlation of histological parameters with SOH of SGT

\begin{tabular}{|c|c|c|c|c|c|c|}
\hline \multirow{2}{*}{\multicolumn{2}{|c|}{ Histological parameters }} & \multicolumn{3}{|c|}{$\mathrm{SOH}$} & \multirow[b]{2}{*}{$x^{2}$} & \multirow[b]{2}{*}{$p$ value } \\
\hline & & Mild (\%) & Moderate (\%) & Intense (\%) & & \\
\hline \multirow[t]{3}{*}{ PR color } & Reddish & 60.0 & 0.0 & 40 & & \\
\hline & Yellowish-red & 0.0 & 0.0 & 100 & 6.240 & 0.044 \\
\hline & Greenish-yellow & 0.0 & 0.0 & 100 & & \\
\hline \multirow[t]{3}{*}{ PR intensity } & Mild & 0.0 & 0.0 & 0.0 & & \\
\hline & Moderate & 0.0 & 0.0 & 0.0 & - & - \\
\hline & Intense & 23.1 & 0.0 & 76.9 & & \\
\hline \multirow[t]{3}{*}{ PR pattern } & NS & 0.0 & 0.0 & 100.0 & & \\
\hline & $F$ & 50.0 & 0.0 & 50.0 & 2.136 & 0.344 \\
\hline & D & 28.6 & 0.0 & 71.4 & & \\
\hline \multirow[t]{3}{*}{$A B$ intensity } & Mild & 0.0 & 0.0 & 0.0 & & \\
\hline & Moderate & 0.0 & 0.0 & 100.0 & 0.709 & 0.400 \\
\hline & Intense & 27.3 & 0.0 & 72.7 & & \\
\hline \multirow[t]{3}{*}{ AB pattern } & NS & 0.0 & 0.0 & 0.0 & & \\
\hline & $F$ & 50.0 & 0.0 & 50.0 & 0.965 & 0.326 \\
\hline & D & 18.2 & 0.0 & 81.8 & & \\
\hline \multirow[t]{3}{*}{ SNO intensity } & Mild & 0.0 & 0.0 & 0.0 & & \\
\hline & Moderate & 0.0 & 0.0 & 0.0 & - & - \\
\hline & Intense & 23.1 & 0.0 & 76.9 & & \\
\hline \multirow[t]{3}{*}{ SNO pattern } & NS & 0.0 & 0.0 & 0.0 & & \\
\hline & $F$ & 0.0 & 0.0 & 0.0 & - & - \\
\hline & D & 23.1 & 0.0 & 76.9 & & \\
\hline \multirow[t]{3}{*}{ PAS intensity } & Mild & 0.0 & 0.0 & 100.0 & & \\
\hline & Moderate & 16.7 & 0.0 & 83.3 & 1.546 & 0.462 \\
\hline & Intense & 40.0 & 0.0 & 60.0 & & \\
\hline \multirow[t]{3}{*}{ PAS pattern } & NS & 0.0 & 0.0 & 0.0 & & \\
\hline & $F$ & 0.0 & 0.0 & 0.0 & - & - \\
\hline & D & 23.1 & 0.0 & 76.9 & & \\
\hline
\end{tabular}

Chi-squared test, $p$-value $<0.05$-statistically significant

Table 4: Comparison of correlation of SOH with recurrence of SGT

\begin{tabular}{lcccccc}
\hline $\begin{array}{l}\text { Salivary } \\
\text { gland } \\
\text { tumors }\end{array}$ & SOH & Recurrence & $\begin{array}{c}\text { No } \\
\text { recurrence }\end{array}$ & Total & $x^{2}$ & $p$ value \\
\hline (13 & 3 & 7 & 0 & 7 & & \\
Samples) & 2 & 1 & 2 & 3 & 6.74 & $\mathbf{0 . 0 3 4 ^ { * }}$ \\
& 1 & 1 & 2 & 3 & & \\
\hline
\end{tabular}

Chi-squared test, $p$-value $<0.05$-statistically ${ }^{*}$ significant

In the current study, a total of 53 OSMF cases were analyzed by four differential stains to ascertain the $\mathrm{SOH}$. It was observed that intensity $(p=0.008)$ and pattern $(p=0.010)$ of Alcian blue staining and intensity of safranin O stain $(p=0.003)$ significantly correlated with $\mathrm{SOH}$ (Fig. 10). Picrosirius red polarizing color reddish and yellowish red $(p=0.002)$ significantly correlated with $\mathrm{SOH}$ enabling to distinguish early and advanced OSMF (Fig. 11). Also, histological features of the atrophic epithelium $(p=0.001)$ and muscle degeneration ( $p=0.000)$ showed a strong statistical correlation with $\mathrm{SOH}$ indicating advanced disease.
Table 5: Descriptive features of benign soft tissue lesions

\begin{tabular}{lcc}
\hline Characteristics & Frequency & Percentage (\%) \\
\hline Age-groups & & \\
$21-40$ & 7 & 29.2 \\
$41-60$ & 11 & 45.8 \\
$>61$ & 6 & 25 \\
Gender & & \\
$\quad$ Male & 11 & 45.8 \\
Female & 13 & 54.2 \\
Subgroups & & \\
$\quad$ Reactive lesions & 13 & 54.2 \\
Tumor/tumor-like lesions & 11 & 45.8 \\
Site & & \\
$\quad$ Mandibular vestibule & 3 & 12.5 \\
Maxillary sinus & 3 & 12.5 \\
Corner of mouth & 1 & 4.2 \\
Buccal mucosa & 6 & 25.0 \\
Labial mucosa & 1 & 4.2 \\
Gingiva & 3 & 12.5 \\
Maxillary vestibule & 5 & 20.8 \\
Tongue & 2 & 8.3 \\
\hline
\end{tabular}






Figs 4A to O: Photomicrographs of BST lesions. (A to C) Irritational fibroma showing mild staining for PAS and Alcian blue, and intense staining for safranin O ( $\times 100)$; (D to F) Traumatic neuroma showing mild staining for PAS and Alcian blue, and intense staining safranin O ( $\times 100)$; (G to I) Neurilemmoma showing mild staining for PAS, Alcian blue, and safranin $\mathrm{O}(\times 100)$; $(\mathrm{J}$ to $\mathrm{L})$ Myxoid neurofibroma showing intense staining for PAS, Alcian blue, and safranin $\mathrm{O}(\times 100)$; ( $\mathrm{M}$ to $\mathrm{O})$ Nodular fasciitis showing mild staining for PAS, intense staining for Alcian blue and safranin $\mathrm{O}(\times 100)$ 

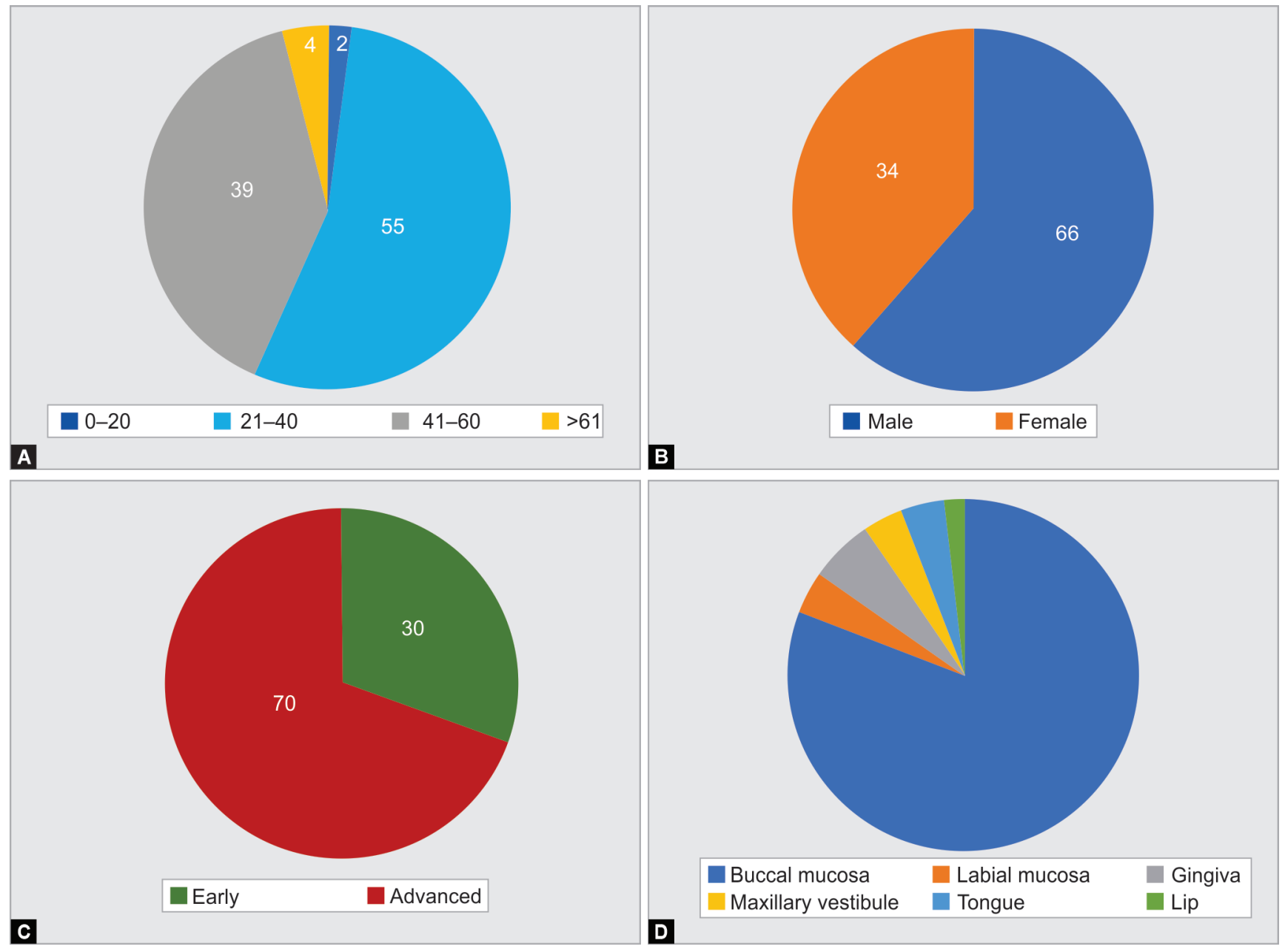

Figs 5 A to D: Descriptive features of OSMF cases

Table 6: Comparison of correlation of general parameters with SOH of OSMF

\begin{tabular}{|c|c|c|c|c|c|c|}
\hline \multirow[b]{2}{*}{ Clinical parameters } & \multicolumn{6}{|c|}{$\mathrm{SOH}$} \\
\hline & & Mild (\%) & Moderate (\%) & Intense (\%) & $x^{2}$ & $p$ value \\
\hline \multirow[t]{6}{*}{ Site } & Buccal mucosa & 16.3 & 7.0 & 76.7 & \multirow{6}{*}{10.582} & \multirow{6}{*}{0.391} \\
\hline & Labial mucosa & 0.0 & 0.0 & 100.0 & & \\
\hline & Gingiva & 0.0 & 33.3 & 66.7 & & \\
\hline & Maxillary vestibule & 0.0 & 0.0 & 100.0 & & \\
\hline & Tongue & 0.0 & 0.0 & 100.0 & & \\
\hline & Lip & 100.0 & 0.0 & 0.0 & & \\
\hline \multirow[t]{4}{*}{ Atrophic epithelium } & Nil & 55.6 & 11.1 & 33.3 & \multirow{4}{*}{23.793} & \multirow{4}{*}{0.001} \\
\hline & Mild & 25.0 & 8.3 & 66.7 & & \\
\hline & Moderate & 0.0 & 22.2 & 77.8 & & \\
\hline & Severe & 0.0 & 0.0 & 100.0 & & \\
\hline \multirow[t]{2}{*}{ Muscle degeneration } & Absent & 38.9 & 16.7 & 44.4 & \multirow{2}{*}{17.045} & \multirow{2}{*}{0.000} \\
\hline & Present & 2.9 & 2.9 & 94.3 & & \\
\hline
\end{tabular}


Table 7: Comparison of correlation of histological parameters with SOH of OSMF

\begin{tabular}{|c|c|c|c|c|c|c|}
\hline \multirow{2}{*}{\multicolumn{2}{|c|}{ Histological parameters }} & \multicolumn{3}{|c|}{$\mathrm{SOH}$} & \multirow[b]{2}{*}{$x^{2}$} & \multirow[b]{2}{*}{$p$ value } \\
\hline & & Mild (\%) & Moderate (\%) & Intense (\%) & & \\
\hline \multirow[t]{3}{*}{ PR color } & Reddish & 0.0 & 0.0 & 100.0 & 13.444 & 0.009 \\
\hline & Yellowish-red & 8.0 & 8.0 & 84.0 & & \\
\hline & Greenish-yellow & 40.0 & 13.3 & 46.7 & & \\
\hline \multirow[t]{3}{*}{ PR intensity } & Mild & 0.0 & 0.0 & 0.0 & 2.765 & 0.251 \\
\hline & Moderate & 0.0 & 8.3 & 91.7 & & \\
\hline & Intense & 19.5 & 7.3 & 73.2 & & \\
\hline \multirow[t]{3}{*}{ PR pattern } & NS & 0.0 & 0.0 & 0.0 & 0.608 & 0.738 \\
\hline & $\mathrm{F}$ & 0.0 & 0.0 & 100.0 & & \\
\hline & D & 15.7 & 7.8 & 76.5 & & \\
\hline \multirow[t]{3}{*}{$A B$ intensity } & Mild & 42.9 & 14.3 & 42.9 & 13.926 & 0.008 \\
\hline & Moderate & 0.0 & 0.0 & 100.0 & & \\
\hline & Intense & 5.4 & 5.4 & 89.2 & & \\
\hline \multirow[t]{3}{*}{ AB pattern } & NS & 0.0 & 0.0 & 0.0 & 9.284 & 0.010 \\
\hline & $F$ & 35.3 & 11.8 & 52.9 & & \\
\hline & D & 5.6 & 5.6 & 88.9 & & \\
\hline \multirow[t]{3}{*}{ SNO intensity } & Mild & 0.0 & 0.0 & 0.0 & 11.691 & 0.003 \\
\hline & Moderate & 100.0 & 0.0 & 0.0 & & \\
\hline & Intense & 11.8 & 7.8 & 80.4 & & \\
\hline \multirow[t]{3}{*}{ SNO pattern } & NS & 0.0 & 0.0 & 0.0 & - & - \\
\hline & $F$ & 0.0 & 0.0 & 0.0 & & \\
\hline & D & 15.1 & 7.5 & 77.4 & & \\
\hline \multirow[t]{3}{*}{ PAS intensity } & Mild & 23.1 & 7.7 & 69.2 & 6.070 & 0.194 \\
\hline & Moderate & 25.0 & 12.5 & 62.5 & & \\
\hline & Intense & 0.0 & 5.3 & 94.7 & & \\
\hline \multirow[t]{3}{*}{ PAS pattern } & NS & 0.0 & 0.0 & 0.0 & 3.592 & 0.166 \\
\hline & $F$ & 22.2 & 11.1 & 66.7 & & \\
\hline & D & 7.7 & 3.8 & 88.5 & & \\
\hline
\end{tabular}

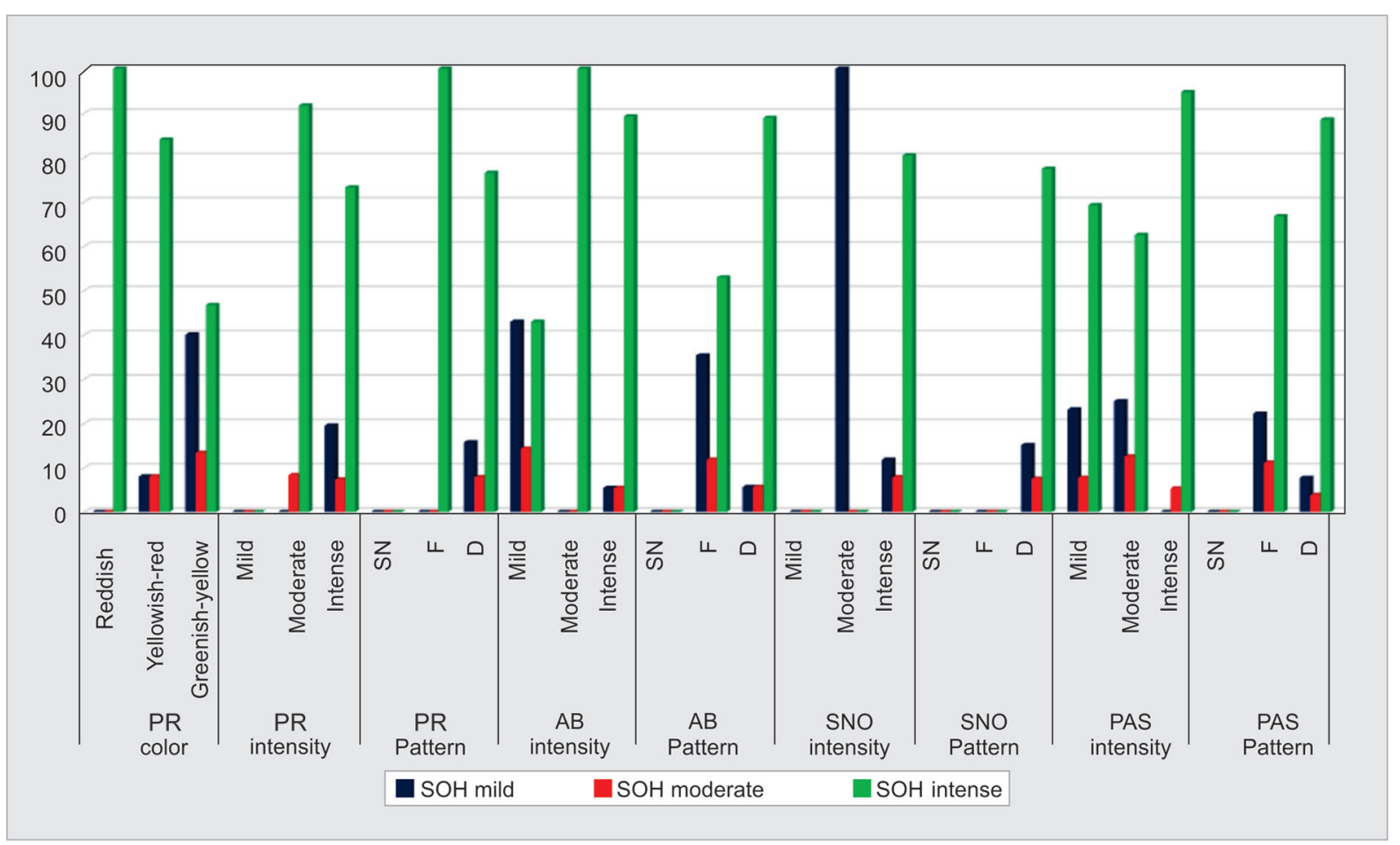

Fig. 6: Comparison of correlation of histological parameters with SOH of OSMF 

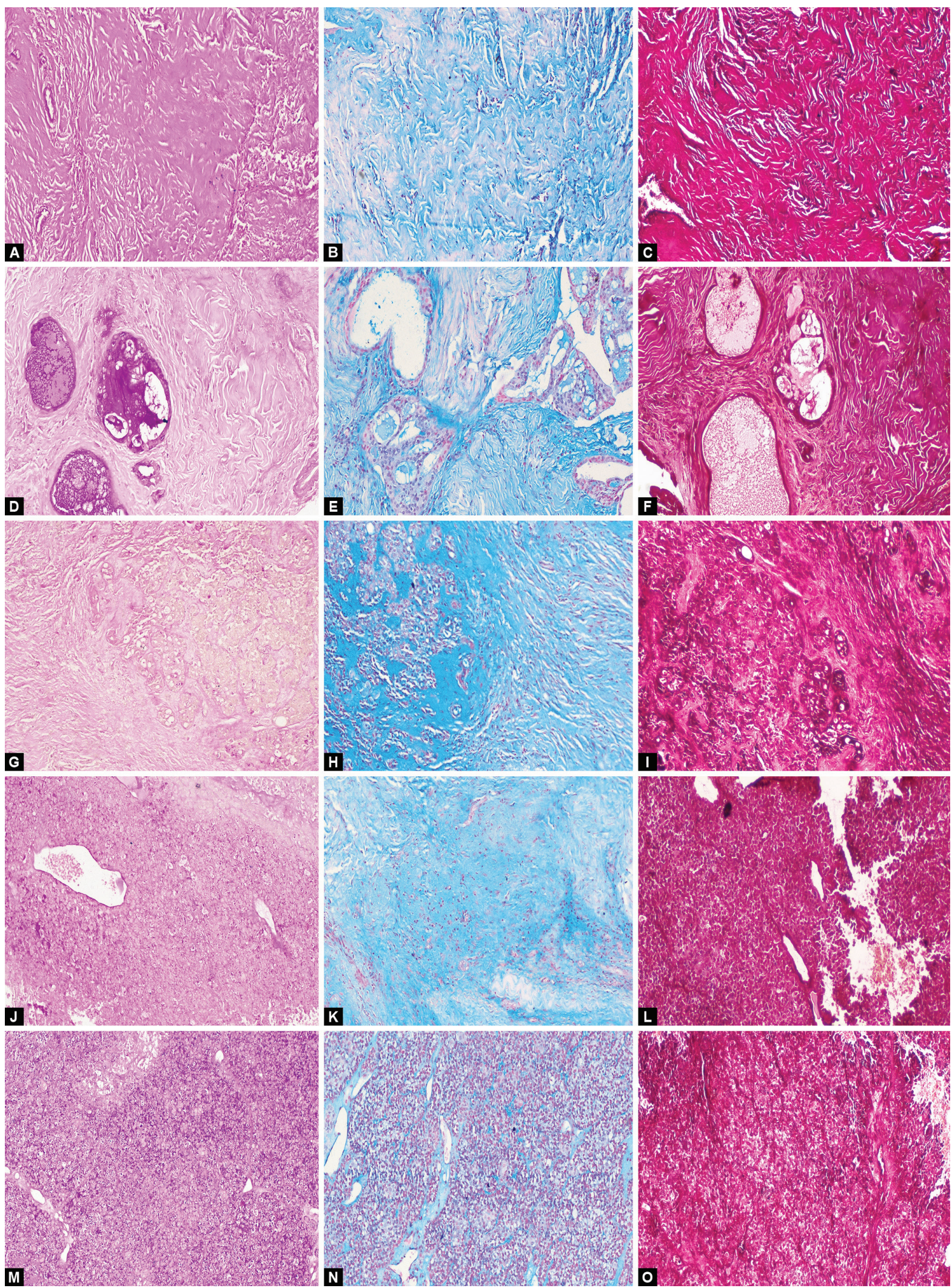

Figs 7A to O: Photomicrographs of SGTs. (A to C) Pleomorphic adenoma showing mild staining for PAS and Alcian blue, and intense staining for safranin O ( $\times 100)$; (D to F) Mucoepidermoid carcinoma showing mild staining for PAS and moderate staining for Alcian blue and safranin O ( $\times 100)$; ( $G$ to I) Carcinoma ex pleomorphic adenoma showing mild staining for PAS, and intense staining for Alcian blue and safranin O ( $\times 100)$; ( $\mathrm{J}$ to $\mathrm{L}$ ) Carcinoma ex pleomorphic adenoma showing intense staining for PAS, Alcian blue, and safranin O ( $\times 100)$; (M to O) Carcinoma ex pleomorphic adenoma showing intense staining for PAS, Alcian blue, and safranin $\mathrm{O}(\times 100)$ 

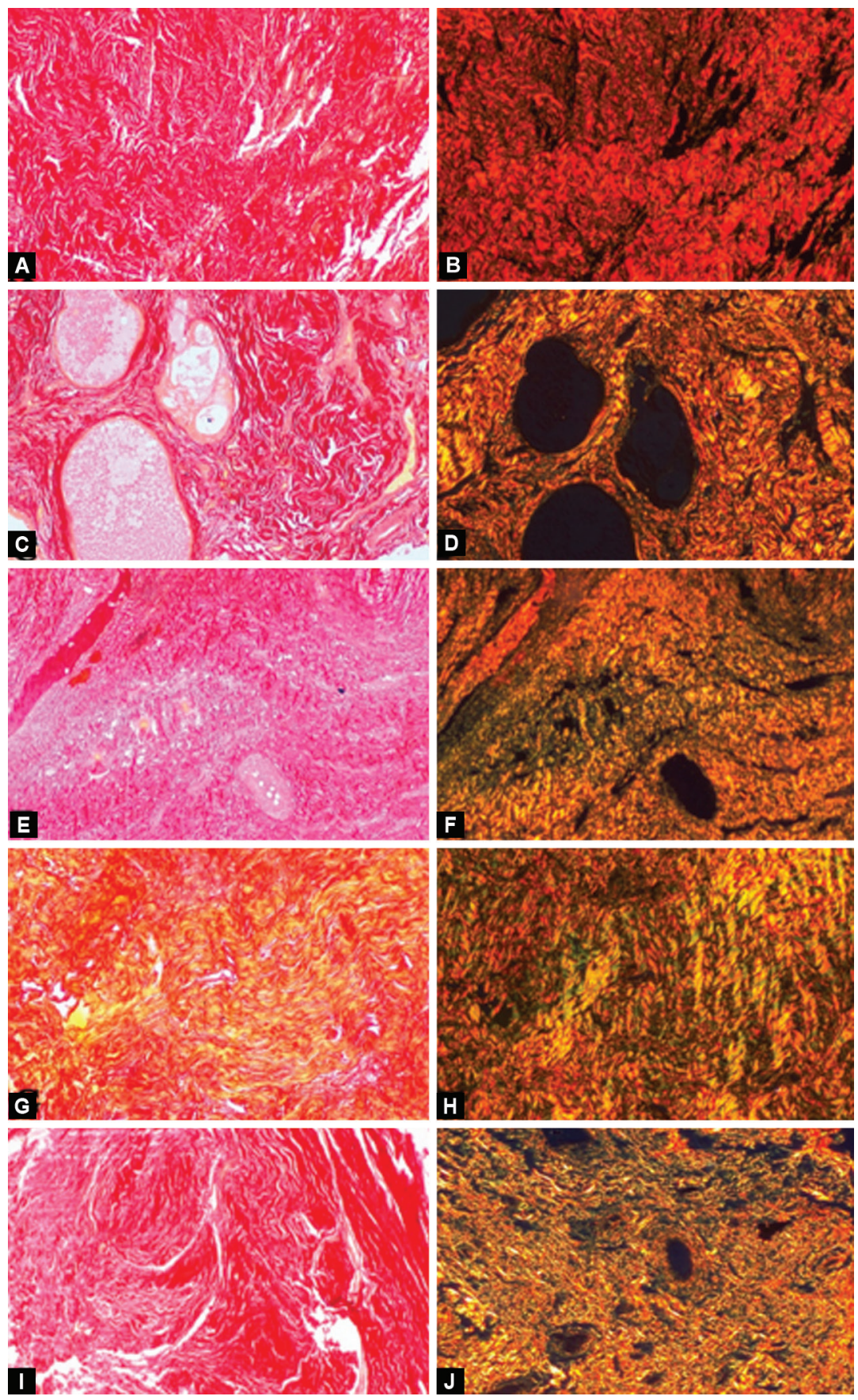

Figs 8 A to J: Picrosirius red stain/polarizing microscopy of SGT. (A and B) Pleomorphic adenoma showing intense staining and reddish collagen fibers ( $\times 100)$; (C and D) Mucoepidermoid carcinoma showing intense staining and yellowish-green collagen fibers ( $\times 100)$; $(E$ and $F)$ Carcinoma ex pleomorphic adenoma showing intense staining and greenish-yellow collagen fibers $(\times 100) ;(G$ and H) Carcinoma ex pleomorphic adenoma showing intense staining and greenish-yellow collagen fibers ( $\times 100)$; ( I and J) Carcinoma ex pleomorphic adenoma showing intense staining and greenish-yellow collagen fibers $(\times 100)$ 

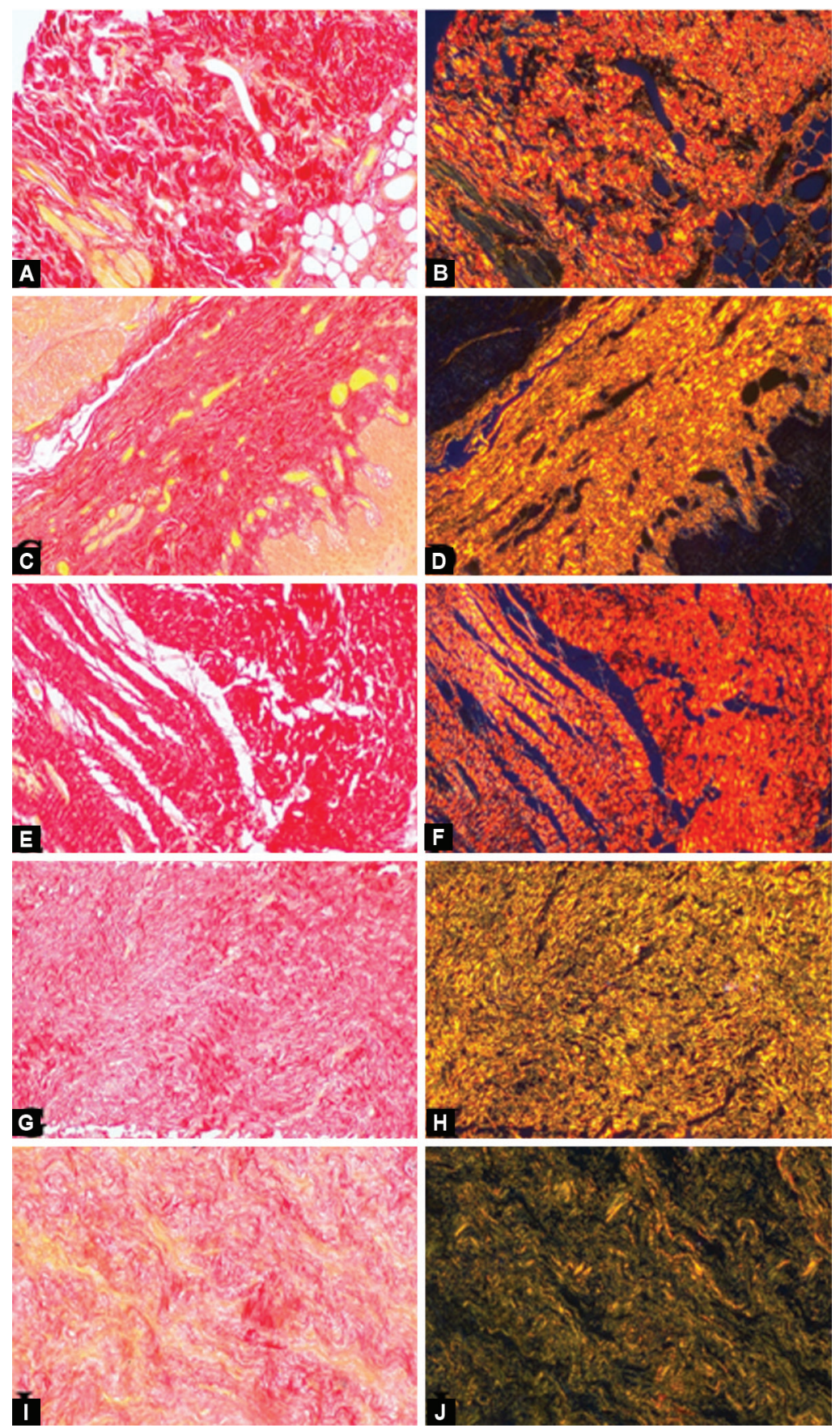

Figs 9A to J: Picrosirius red stain/polarizing microscopy of BST. (A and B) Irritational fibroma showing intense staining and reddish collagen fibers $(\times 100)$; $(C$ and D) Traumatic neuroma showing intense staining and yellowish-red collagen fibers $(\times 100)$; $(E$ and $F)$ Neurilemmoma showing intense staining and reddish collagen fibers $(\times 100) ;(G$ and $H)$ Myxoid neurofibroma showing intense staining and yellowish-green collagen fibers $(\times 100)$; $(I$ and $J)$ Nodular fasciitis showing intense staining and greenish-yellow collagen fibers $(\times 100)$ 




Figs 10A to O: Photomicrographs of OSMF. (A to C) Early OSMF showing mild staining for PAS, Alcian blue, and safranin O ( $\times 100)$; (D to F) Early OSMF showing mild staining for PAS and Alcian blue, and moderate staining safranin O ( $\times 100)$; (G to I) Early OSMF showing mild staining for PAS, Alcian blue, and safranin O $(\times 100)$; ( $\mathrm{J}$ to $\mathrm{L})$ Advanced OSMF showing moderate staining for PAS and intense staining for Alcian blue and safranin $\mathrm{O}$ ( $\times 100)$; (M to O) Advanced OSMF showing intense staining for PAS, Alcian blue, and safranin $\mathrm{O}(\times 100)$ 

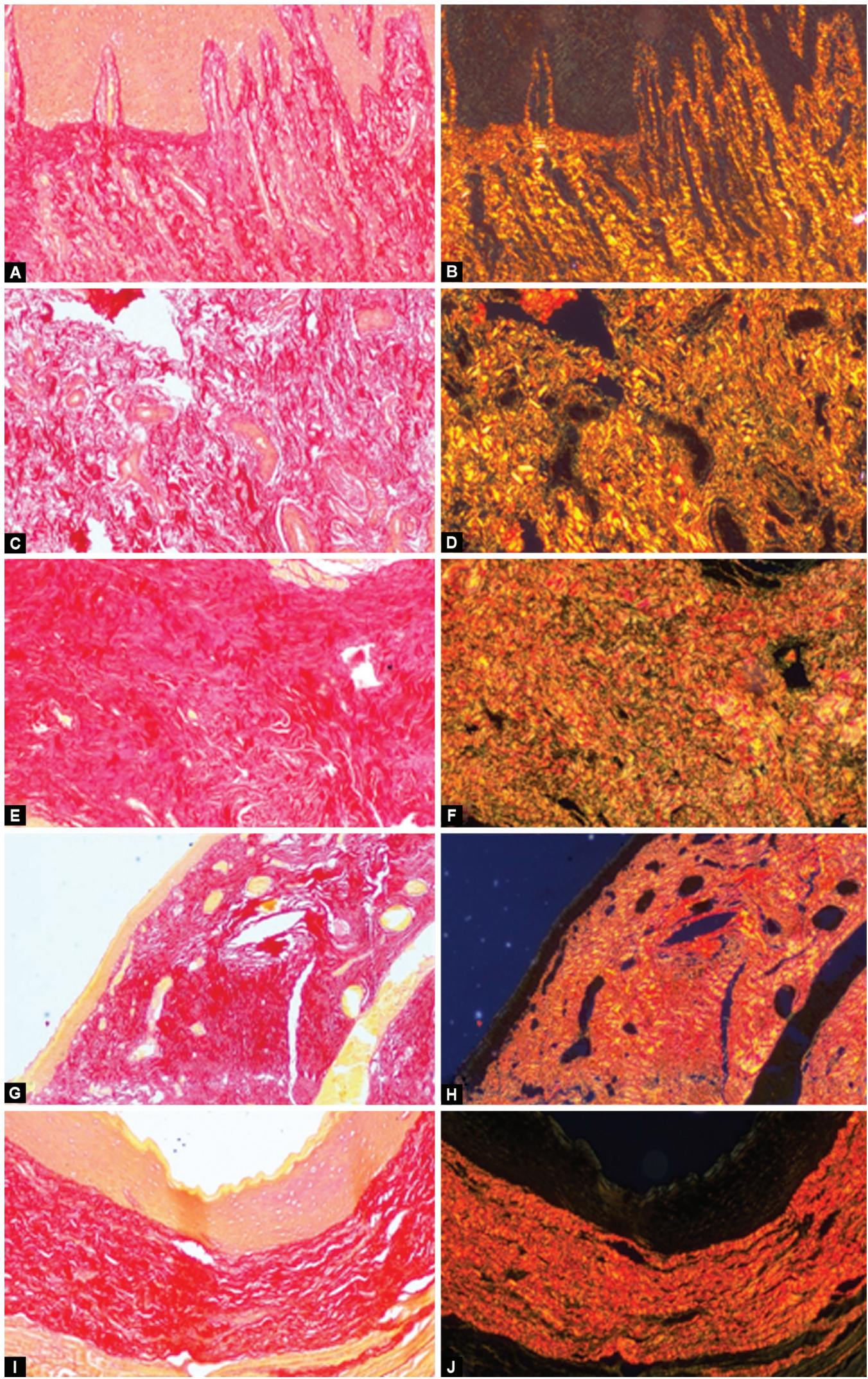

Figs $11 \mathrm{~A}$ to J: Picrosirius red stain/polarizing microscopy of OSMF. (A and B) Early OSMF showing intense staining and yellowish-green collagen fibers ( $\times 100)$; (C and D) Early OSMF showing intense staining and yellowish-green collagen fibers ( $\times 100)$; (E and F) Early OSMF showing intense staining and yellowish-green collagen fibers with patches of red $(\times 100)$; $(\mathrm{G}$ and $\mathrm{H})$ Advanced OSMF showing intense staining and reddish-yellow collagen fibers ( $\times 100)$; (I and J) Advanced OSMF showing intense staining and reddish collagen fibers $(\times 100)$ 
Table 8: Comparison of picrosirius red polarizing microscopy in early and advanced OSMF

\begin{tabular}{lccccc}
\hline Polarizing colors & Early & Advanced & & & \\
\hline OSMF & OSMF & Total & $X^{2}$ & p value \\
\hline Geenish-yellow & 08 & 10 & 18 & 6.087 & $0.04^{*}$ \\
Reddish & 02 & 08 & 10 & & \\
Total & 03 & 22 & 25 & & \\
\hline
\end{tabular}

${ }^{*}$ significant

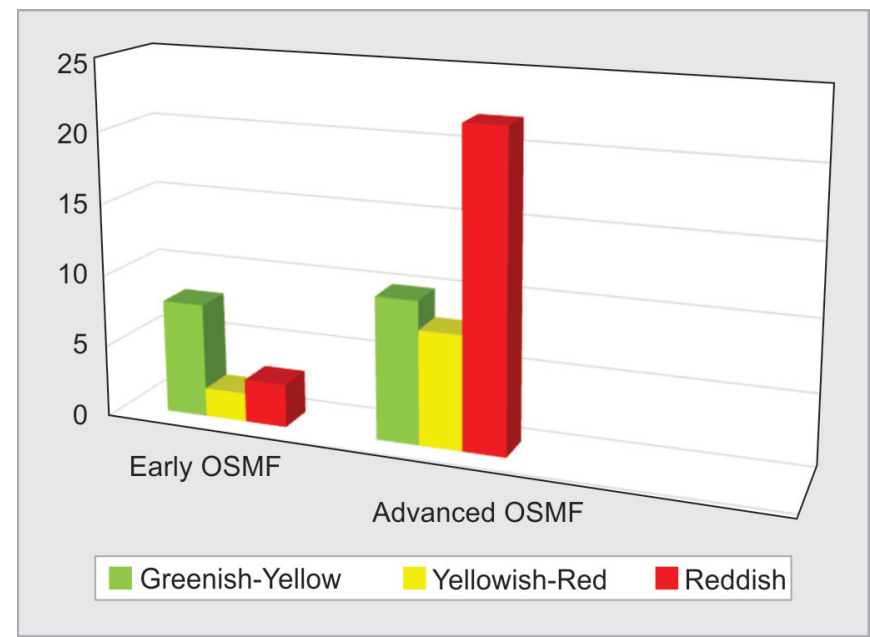

Fig. 12: Difference in picrosirius red polarizing microscopy in early and advanced OSMF

The equilibrium of stromal hyalinization that is preceded by fibrosis is maintained by periods of collagenolytic activity and collagen deposition. The resulting fibrosis is due to an interplay of two pathways; first, TGF- $\beta$ inhibits the transcription enzymes responsible for collagenolysis resulting in decreased collagenolysis. The collagen inhibitor proteins SMAD6 and SMAD7 are downregulated by TGF- $\beta$ ensuing more fibrosis and hyalinization. ${ }^{30,31}$

Usually, the transition of mature to immature collagen fibers indicates aggressive transformation that has been demonstrated in recent studies. ${ }^{32}$ This change can be assessed by picrosirius red polarizing microscopy that would indicate a change in color from reddish to greenish-yellow. In contrast to this, the current study has demonstrated a predominantly birefringent reddish color in advanced OSMF cases and a predominantly greenish-yellow color in early OSMF cases. This could be due to fact that advanced cases show a thicker packing and aggregation of collagen due to fibrosis compared to the loose arrangement in early OSMF cases. ${ }^{33}$ The above finding is supported by two studies by Savita and Naik ${ }^{34}$ and Ashalata et al., ${ }^{35}$ who analyzed OSMF cases by picrosirius red polarizing microscopy and concluded that there was a shift in the color of collagen fibers from greenish-yellow to an orangish-red and red color as the severity of the disease increased. This brands picrosirius red polarizing microscopy a reliable tool in differentiating early and advanced OSMF (Table 8 and Fig. 12).

\section{ConcLusion}

The influence of hyalinization on the biologic behavior of oral lesions is the least explored domain of research. This study is the first of its kind, to correlate the intensity of hyalinization to the biological behavior of OHEOL by using four special stains. Knowledge of the biological behavior of these lesions can help prevent conservative management of lesions that eventually recur or unnecessary aggressive management of lesions. As a result, the surgeons will have a better idea of how to effectively manage OHEOL based on the results of the current study.

\section{ACKnOWledgments}

The Authors thank Dr. Suvi Kanchan for performing the statistical analysis.

\section{References}

1. Saluja T, lyer J. Unmasking the grey zone of hyalinization with a proposed classification of oral hyalinizing lesions. J Interdiscipl Histopathol 2017;5(1):18-21. DOI: 10.5455/jihp.20160821122428.

2. Dicker KT, Gurski LA, Pradhan-Bhatt $S$, et al. Hyaluronan: a simple polysaccharide with diverse biological functions. Acta Biomater 2014;10(4):1558-1570. DOI: 10.1016/j.actbio.2013.12.019.

3. Ropponen K, Tammi M, Parkkinen J, et al. Tumor cell-associated hyaluronan as an unfavourable prognostic factor in colorectal cancer. Cancer Res 1998;58(2):342-347.

4. Bratthauer $G L$. The avidin-biotin complex $(A B C)$ method and other avidin-biotin binding methods. Methods Mol Biol 2010;588:257-270. DOI: 10.1007/978-1-59745-324-0_26.

5. Alturkistani HA, Tashkandi FM, Mohammedsaleh ZM. Histological stains: a literature review and case study. Glob J Health Sci 2015;8(3):72-79. DOI: 10.5539/gjhs.v8n3p72.

6. Hui H, Ma W, Cui J, et al. Periodic acid Schiff staining method for function detection of liver cells is affected by $2 \%$ horse serum in induction medium. Mol Med Rep 2017;16(6):8062-8068. DOI: 10.3892/ mmr.2017.7587.

7. Alibegovic A, Blagus R, Martinez IZ. Safranin O without fast green is the best staining method for testing the degradation of macromolecules in a cartilage extracellular matrix for the determination of the postmortem interval. Forensic Sci Med Pathol 2020;16(2):252-258. DOI: 10.1007/s12024-019-00208-0.

8. Meyerholz DK, Rodgers J, Castilow EM, et al. Alcian blue and Pyronine $\mathrm{Y}$ histochemical stains permit assessment of multiple parameters in pulmonary disease models. Vet Pathol 2009;46(2):325-328. DOI: 10.1354/vp.46-2-325.

9. Rittié L. Method for Picrosirius red-polarization detection of collagen fibers in tissue sections. Methods Mol Biol 2017;1627:395-407. DOI: 10.1007/978-1-4939-7113-8_26.

10. Fletcher CD. Recently characterized soft tissue tumors that bring biologic insight. Mod Pathol 2014;27(1):S98-S112. DOI: 10.1038/ modpathol.2013.172.

11. Kumari K, Ghosh S, Patil S, et al. Expression of type III collagen correlates with poor prognosis in oral squamous cell carcinoma. J Investig Clin Dent 2017;8(4):e12253. DOI: 10.1111/jicd.12253.

12. Cottom HE, Bshena Fl, Speight PM, et al. Histopathological features that predict the recurrence of odontogenic keratocysts. J Oral Pathol Med 2012;41(5):408-414. DOI: 10.1111/j.1600-0714.2011.01113.x.

13. McGavin MD. Factors affecting visibility of a target tissue in histologic sections. Vet Pathol 2014;51(1):9-27. DOI: 10.1177/0300985813506916.

14. Kilcoyne M, Gerlach JQ, Farrell MP, et al. Periodic acid-Schiff's reagent assay for carbohydrates in a microtiter plate format. Anal Biochem 2011;416(1):18-26. DOI: 10.1016/j.ab.2011.05.006.

15. Lattouf $R$, Younes $R$, Lutomski $D$, et al. Picrosirius red staining: a useful tool to appraise collagen networks in normal and pathological tissues. J Histochem Cytochem 2014;62(10):751-758. DOI: 10.1369/0022155414545787.

16. Hyllested JL, Veje K, Ostergaard K. Histochemical studies of the extracellular matrix of human articular cartilage-a review. Osteoarthritis Cartilage 2002;10(5):333-343. DOI: 10.1053/joca.2002.0519. 
17. Dong W, Matsuno YK, Kameyama A. A procedure for Alcian blue staining of mucins on polyvinylidene difluoride membranes. Anal Chem 2012;84(20):8461-8466. DOI: 10.1021/ac301678z.

18. Bobati SS, Patil BV, Dombale VD. Histopathological study of salivary gland tumors. J Oral Maxillofac Pathol 2017;21(1):46-50. DOI: 10.4103/0973-029X.203762.

19. Speight PM, Barrett AW. Salivary glands and saliva. Oral Dis 2002;8:229-240.

20. Auclair PL, Ellis GL. Atypical features in salivary gland mixed tumors: their relationship to malignant transformation. Mod Pathol 1996;9(6):652-657.

21. Weinreb I. Hyalinizing clear cell carcinoma of salivary gland: a review and update. Head Neck Pathol 2013;7(Suppl. 1):S20-S29. DOI: 10.1007/ s12105-013-0466-8.

22. Speight PM, Barrett AW. Salivary gland tumours: diagnostic challenges and an update on the latest WHO classification. Diagn Histopathol 2020;26(4):147-158. DOI: 10.1016/j.mpdhp.2020.01.001.

23. Krane JF, Faquin WC. Salivary gland. In: Cibas ES, Ducatman BS, editors. Cytology diagnostic principles and clinical correlates. 4th ed. Saunders; 2014. p. 299-332.

24. Seethala RR. Basaloid/blue salivary gland tumors. Mod Pathol 2017;30(1):S84-S95. DOI: 10.1038/modpathol.2016.190.

25. Ito FA, Jorge J, Vargas PA, et al. Histopathological findings of pleomorphic adenomas of the salivary glands. Med Oral Patol Oral Cir Bucal 2009;14(2):E57-E61.

26. Masamatti SS, Gosavi AV. Benign soft tissue lesions of oral cavity: a histopathological study. J Clin Biomed Sci 2016;6(3):88-90.

27. Chouaib S, Messai $Y$, Couve $S$, et al. Hypoxia promotes tumor growth in linking angiogenesis to immune escape. Front Immunol 2012;3:21. DOI: 10.3389/fimmu.2012.00021.
28. Auluck A, Rosin MP, Zhang $L$, et al. Oral submucous fibrosis, a clinically benign but potentially malignant disease: report of 3 cases and review of the literature. J Can Dent Assoc 2008;74(8): 735-740.

29. Kamath VV. The nature of collagen in oral submucous fibrosis: a systematic review of the literature. Saudi J Oral Sci 2014;1(2):57-64. DOI: 10.4103/1658-6816.138461.

30. Pakyari M, Farrokhi A, Maharlooei MK, et al. Critical role of transforming growth factor beta in different phases of wound healing. Adv Wound Care (New Rochelle) 2013;2(5):215-224. DOI: 10.1089/wound.2012.0406.

31. Miyazawa K, Miyazono K. Regulation of TGF- $\beta$ family signaling by inhibitory Smads. Cold Spring Harb Perspect Biol 2017;9(3):01-25. DOI: 10.1101/cshperspect.a022095.

32. Arun Gopinathan P, Kokila G, Jyothi M, et al. Study of collagen birefringence in different grades of oral squamous cell carcinoma using Picrosirius red and polarized light microscopy. Scientifica (Cairo) 2015;2015:01-07. DOI: 10.1155/2015/802980.

33. Tom A, Baghirath V, Krishna B, et al. Ultrastructural changes of collagen in different histopathological grades of oral submucous fibrosis. J Pharm Bioallied Sci 2019;11(Suppl. 2):S309-S313. DOI: 10.4103/JPBS. JPBS_20_19.

34. Thakkannavar SS, Naik VV. Histochemical and immunohistochemical analysis of collagen fibers and microvascular density in various grades of oral submucous fibrosis. Iran J Pathol 2019;14(2):127-134. DOI: 10.30699/IJP.14.2.127.

35. Ashalata G, Baghirath PV, Krishna AB, et al. Quantitative and qualitative analysis of collagen in oral submucous fibrosis. J NTR Univ Health Sci 2012;1(2):99-105. DOI: 10.4103/2277-8632.98350. 